\title{
Bestimmt die wirtschaftliche Dynamik der Städte die Intensität der Einkommenspolarisierung?
}

Resultate für deutsche Stadtregionen

\section{Thesen und Fragen}

Die großen europäischen Städte unterliegen seit den 1970er Jahren einem sozioökonomischen Strukturwandel, der sowohl das Städtesystem als auch die innere Struktur der Städte tangiert. Stadtentwicklung ist heute nicht mehr überall identisch mit Wachstum: Einigen weiterhin prosperierenden Städten steht eine wachsende Zahl schrumpfender Städte gegenüber. Ein einheitliches Modell von Stadtentwicklung, das auf Zunahme von Bevölkerung und Arbeitsplätzen sowie steigenden Realeinkommen beruhte, existiert zumindest in den OECD-Ländern nicht mehr. Die Pfade der Stadtentwicklung werden nicht nur zwischen den Städten unterschiedlicher, sondern auch innerhalb der Städte vergrößern sich die Differenzen zwischen den Lebenslagen und Lebenschancen ihrer Bewohner. Angesichts dieser Tendenzen muss man daher von einer doppelten Spaltung sprechen: einer Spaltung zwischen den Städten und einer Spaltung innerhalb der Städte.

Mit einem wachsenden Anteil von Dienstleistungstätigkeiten auf dem Arbeitsmarkt - so die These - nimmt die Einkommensungleichheit zu. Entweder wird ein großer Teil der Arbeitskräfte, die früher Fabrikarbeiter waren, aus dem Arbeitsmarkt in die Dauerarbeitslosigkeit abgedrängt - oder aber es wird eine Vollbeschäftigung angestrebt, die nur dann annähernd erreicht werden kann, wenn die Regulierungen von Arbeitsmarkt und Gewerbe einen größeren Niedriglohnsektor zulassen und jene neue Schicht in der Erwerbstätigkeit entsteht, die bisher in deutschen Städten keine große Rolle gespielt hat: diejenigen, die voll arbeiten, aber von ihrer Arbeit nicht leben können, die »working poor «. Von Arbeitslosigkeit und Niedriglohn sind insbesondere die älteren Arbeitnehmer und die gering Qualifizierten betroffen, wozu viele Migranten zählen.

Die zunehmende Tertiarisierung schafft Potentiale für eine höhere Lohndifferenzierung. ${ }^{1}$ Einfache Dienstleistungen mit geringen Möglichkeiten für Produktivitätssteigerungen nehmen nur dann zu, wenn ihre Entlohnung relativ zur Gesamteinkommensentwicklung zurückbleibt. Hochwertige Dienstleistungen können dagegen auch bei steigenden Löhnen expandieren, da Lohnkostenerhöhungen durch Produktivitätssteigerungen kompensiert werden können. Im Tertiarisierungsprozess entstehen dann parallel sowohl relativ schlecht entlohnte einfache Dienstleistungs-

1 Baumol 1967; Scharpf 1986; Häußermann, Siebel 1995.

Leviathan, 40. Jg., 3/2012, S. 371 398 
arbeitsplätze (Gesundheitssektor, Gastronomie etc.) als auch hoch produktive und hoch entlohnte Dienstleistungsarbeitsplätze (etwa im Finanzsektor). Im Übergang von der Industrie- zur Dienstleistungsgesellschaft nimmt dadurch die Lohnspreizung $\mathrm{zu},{ }^{2}$ zumindest so lange, wie es eine hohe Arbeitslosigkeit gibt.

In einer schon 1988 publizierten Studie haben die amerikanischen Ökonomen Harrison und Bluestone gezeigt, dass der Abbau von Arbeitsplätzen in der Industrie und das Wachstum von Dienstleistungen zu einer Veränderung der Einkommensverteilung führt: Die Anteile der besonders gut und die Anteile der besonders wenig verdienenden Beschäftigten hatten seit Mitte der 1970er Jahre in den USA zugenommen - die Anteile des mittleren Segments waren entsprechend geschrumpft. Mit der Veränderung in der Einkommensverteilung verbanden die Autoren die These, dass Deindustrialisierung und Tertiarisierung zu einer Polarisierung der Einkommensstruktur führe. In den von der Deindustrialisierung betroffenen Regionen, in denen die Industrie mit ihren geringeren Lohndifferenzialen ihre stabilisierende Funktion besonders stark einbüßt, zeigte sich daher auch eine besonders ausgeprägte Einkommenspolarisierung. ${ }^{3}$

Die Thesen der zunehmenden Einkommenspolarisierung sind von der Stadtforschung aufgegriffen worden. So hat Saskia Sassen in ihrem Buch »The Global City ${ }^{4}$ die Polarisierung der Einkommen für New York nachgezeichnet und als charakteristisch für solche Städte bezeichnet, in denen sich die Zentralen global agierender Unternehmen und die daran angelagerten Dienstleistungsunternehmen konzentrieren. Sassen stellte einen direkten Zusammenhang zwischen der wachsenden Zahl von hoch qualifizierten und sehr gut verdienenden Beschäftigten in den Bereichen des Finanzwesens, der Rechtsberatung und der High-Tech-Entwicklung einerseits und der steigenden Zahl von gering qualifizierten und schlecht bezahlten Dienstleistungsarbeitern im Bereich von Reinigung, Gastronomie, Transport und Bewachung her, bei denen sie sogar sinkende Reallöhne beobachtete. Diese Polarisierung bringe einen wachsenden Hochlohn- und einen wachsenden Niedriglohnsektor hervor. ${ }^{5}$

Die Vermutung, dass die Einkommenspolarisierung vor allem ein Kennzeichen der Entwicklung in den großen Städten sei, hat auch in Europa zu einer Fülle von Studien zur Analyse der Veränderung der großstädtischen Sozialstrukturen geführt. Die meisten dieser Studien, beispielsweise zu Oslo ${ }^{6}$, Amsterdam und Rotterdam ${ }^{7}$, zu Helsinki ${ }^{8}$ oder zu Zürich ${ }^{9}$, beruhen allerdings auf einer schmalen empirischen

2 Blau 1980.

3 Harrison, Bluestone 1988.

4 Sassen 2001.

5 Sassen 1994.

6 Wessel 2000.

7 Burger, Musterd 2002.

8 Vaattovaara, Kortteinen 2003.

9 Kroll-Schretzenmayr et al. 2005. 
Basis. Im Ergebnis zeigen die Analysen ein eher uneinheitliches Bild. Insbesondere regionalvergleichende Ergebnisse fehlen.

Auf einer breiteren empirischen Basis stehen dagegen die Analysen von Chris Hamnett zu den Entwicklungen in London. Seine Auswertungen zeigen, dass in der wohl den amerikanischen Großstädten ähnlichsten europäischen Stadt keine scharfe Polarisierung stattfindet. Zwar ist auch der Arbeitsmarkt in London von einem dynamischen Wachstum des Dienstleistungsbereichs gekennzeichnet, das zu einer stärkeren Spreizung der Einkommensverteilung führt, allerdings ist es nicht von Einkommensverlusten der niedrigen Einkommen begleitet, denn auch diese verzeichnen prozentuale Zuwächse. Es nimmt also zwar die Ungleichheit zu, aber gleichzeitig erhöhen sich alle Einkommen. ${ }^{10}$ Bezeichnet man die Sassen-These als eine absolute Polarisierung, so könnte man bei den europäischen Befunden von einer relativen Polarisierung sprechen.

Größere Ungleichheit zwischen den Städten und größere Ungleichheit in den Städten - diese Befunde weichen von den seit Beginn der Industrialisierung gewohnten Mustern der Stadtentwicklung ab, sodass die Diagnose von einer »Krise der Städte « ${ }^{11}$ gerechtfertigt erscheint. Mit »Krise der Städte « ist vor allem eine Integrationskrise gemeint, die sich aus der Verschärfung und Überlagerung der genannten Ungleichheiten und den schwindenden Möglichkeiten der Stadtpolitik ergibt, diese Entwicklung so zu steuern, dass soziale Integration und Kohäsion gewährleistet bleiben. Die Krise besteht darin, dass die ökonomischen, kulturellen und sozialen Fragmentierungen und Diskriminierungen, die durch den Bruch mit dem althergebrachten Stadtentwicklungsmodell entstehen, nicht mehr in einem übergreifenden Zusammenhang von Institutionen der sozialen und ökonomischen Inklusion aufgefangen und ausbalanciert werden können, der sich auch aus städtischer Ausgleichspolitik auf der Grundlage einer politischen Solidarität der Stadtbürger ergibt.

Die Städte sind mit der Industrialisierung groß geworden. Manche Städte, die als solche erst mit der Industrie entstanden sind, beispielsweise im Ruhrgebiet, erlebten im Zuge der Deindustrialisierung einen besonders starken ökonomischen Niedergang. Durch Betriebsschließungen und Standortverlagerungen gehen Arbeitsplätze verloren, vor allem jüngere und qualifizierte Bewohner verlassen die Städte, weil sie sich in anderen Regionen eine bessere berufliche Zukunft versprechen. Solche Wanderungen gab es in den alten Bundesländern bereits seit den 1960er Jahren, sie wurden als "Süd-Nord-Gefälle" thematisiert. ${ }^{12}$ Seit der deutschen Wiedervereinigung wird dieses Gefälle durch ein West-Ost-Gefälle überlagert, ${ }^{13}$ weil der Deindustrialisierungsprozess in den neuen Bundesländern zu einer Erosion der ökonomischen und demografischen Basis der Städte geführt hat.

10 Hamnett 2003.

11 Heitmeyer et al. 1998.

12 Friedrichs et al. 1986.

13 Gornig, Häußermann 1998.

Leviathan, 40. Jg., 3/2012 
Wenn Städte über einen gewissen Zeitraum Arbeitsplätze und Bevölkerung verloren haben, kann diese Entwicklung in eine kumulative Abwärtsentwicklung münden, die den Typus der schrumpfenden Stadt charakterisiert.

- Überdurchschnittlicher Einwohnerrückgang durch Geburtendefizit und fehlende Zuwanderung haben eine zunehmende Überalterung zur Folge.

- Rückgang der Zahl der Arbeitsplätze, überdurchschnittliche Arbeitslosigkeit, niedrige Erwerbsquote, rückläufige Realeinkommen und geringe Kaufkraft sind Symptome eines gesellschaftlichen Schrumpfens.

- Rückgang der Steuereinnahmen erschwert die Aufrechterhaltung der Infrastrukturausstattung - der Abbau insbesondere im Jugend- und Bildungsbereich macht eine Stadt dann immer weniger attraktiv gerade für die jüngeren Familien.

Auf der anderen Seite stehen Städte, die Knotenpunkte in den internationalen Netzen der ökonomischen Verflechtung sind, in denen die Zahl der Arbeitsplätze und daher auch die Bevölkerungszahl nach wie vor zunehmen. Das sind vor allem Städte, die keine "altindustrielle " Entwicklung hinter sich haben, sondern erst zu Beginn des 20. Jahrhunderts - und dann bereits auf einer anderen technologischen Grundlage als der Kohle-, Eisen- und Schwerindustrie - in eine industrielle Entwicklung gelangten. Die politische Teilung Deutschlands hat außerdem das Wachstum moderner Industrien und hochwertiger Dienstleistungen in einige westdeutsche Städte gelenkt, die immer noch von den Effekten der Teilung Deutschlands profitieren. Im Folgenden wollen wir einige Indikatoren präsentieren, die zeigen, dass sich die Schere zwischen den Städten in Deutschland öffnet.

Die Ungleichheit in der demografischen wie ökonomischen Entwicklung der Städte ist empirisch auch in Deutschland sehr gut belegt. ${ }^{14}$ Systematische statistische Analysen zur Entwicklung der Einkommensstrukturen innerhalb der Städte fehlen dagegen bislang. Ein wesentlicher Grund dafür ist die unzureichende Datenbasis. ${ }^{15}$ Zwar bemühen sich immer mehr Städte, einen Überblick zur Einkommenssituation ihrer Haushalte zu gewinnen. ${ }^{16}$ Dabei wählen die Städte allerdings zumeist unterschiedliche empirische Ansätze. Eine vergleichende Analyse ist daher auf dieser Basis nicht möglich.

Im vorliegenden Beitrag wird nun eine einheitliche Datenbasis für den Vergleich der Einkommenspolarisierung innerhalb der Stadtregionen verwendet. Die Datenbasis liefern regionale Informationen aus dem Sozio-oekonomischen Panel (SOEP). ${ }^{17}$ Um eine Einschätzung der statistischen Signifikanz der Ergebnisse zu ermöglichen, verwenden wir dabei erstmals verschiedene Polarisationsindizes auf

14 Bade 2006; Geppert, Gornig 2006; Gatzweiler et al. 2009.

15 Gornig 2005.

16 Ähnelt et al. 2009

17 Erste Auswertungen mit dieser Datenbasis erfolgen im Rahmen des Ressortforschungsvorhabens »Trends und Ausmaß der Polarisierung in deutschen Städten «. Bundesministerium für Verkehr, Bau und Stadtentwicklung und Bundesinstitut für Bau-, Stadt- und Raumforschung, Reihe Forschungen 137. 
regionaler Ebene. Die empirische Analyse soll dabei Anhaltspunkte für die Beantwortung folgender beider Fragen geben:

- Ist Einkommenspolarisierung ein Phänomen großstädtischer Regionen oder ist sie ein generelles Kennzeichen der derzeitigen gesellschaftlichen Entwicklung?

- Variiert das Ausmaß der Einkommenspolarisierung danach, wie intensiv und vor allem wie dienstleistungsgeprägt die wirtschaftliche Dynamik einer Stadtregion ist?

\section{Daten und Methoden}

\section{Datengrundlage}

Das Sozio-oekonomische Panel ist eine repräsentative Längsschnitterhebung von sozioökonomischen und demografischen Merkmalen zur Erforschung der Lebensbedingungen in der Bevölkerung. ${ }^{18}$ Die Daten des SOEP werden in den alten Bundesländern bei denselben Haushalten und Personen jährlich seit Mitte der 1980er Jahre erhoben. Die neuen Bundesländer sind seit der deutschen Vereinigung im Jahr 1990 integriert. Infolge der umfangreichen Zuwanderungen aus dem Ausland Ende der 1980er bis zu Beginn der 1990er Jahre wurden seit 1994/95 Zuwandererhaushalte in einer eigenen Stichprobe ergänzend gezogen. Im Laufe der Jahre folgten weitere Zusatzstichproben, insbesondere eine Verdoppelung des Stichprobenumfangs im Jahre 2000 und die Integration einer Hocheinkommensstichprobe im Jahr 2002.

Der Stichprobenumfang des SOEP variiert zudem von Jahr zu Jahr infolge der Mortalität und der neu aufgenommenen Personen und Haushalte. Im Jahr 1984 wurden 5.921 Haushalte mit 12.290 befragten Personen erfasst; nach Ausweitung der Stichprobe im Jahr 2000 hat sich der Umfang im Jahr 2003 mit 12.061 Haushalten und 22.611 befragten Personen gegenüber dem Ausgangszeitpunkt etwa verdoppelt. Langfristig wird ein dauerhafter Stichprobenumfang von ca. 11.000 Haushalten angestrebt. Insgesamt wurden im gesamten Zeitraum seit 1984 mehr als 50.000 Personen kontaktiert.

Diese Erweiterungen des SOEP ergeben die Möglichkeit, auf Basis einer größeren Fallzahl kleine Teilgruppen der Bevölkerung besser zu analysieren, was insbesondere regionalen Differenzierungen zugute kommt. Die Fallzahlen ab 2002 bis 2006 finden sich in Tabelle 1.

18 Die Feldarbeit erfolgt durch TNS Infratest Sozialforschung, München. Für weitere Informationen zum SOEP vgl. Goebel et al. 2008; DIW 2008; siehe auch http://www.diw.de/ soep. 
Tabelle 1: Stichprobengröße des SOEP 2002 bis 2006

\begin{tabular}{|c|c|}
\hline \multicolumn{2}{|c|}{ Stichprobengröße SOEP 2002-2006 (Haushalte) } \\
\hline Jahr & Anzahl Haushalte \\
\hline 2002 & 11.468 \\
2003 & 11.150 \\
2004 & 10.892 \\
2005 & 10.561 \\
2006 & 10.134 \\
\hline
\end{tabular}

Quelle: SOEP.v 26, Stichproben A-F, eigene Berechnungen.

Zur Beschreibung der Einkommenssituation nutzen wir das offen abgefragte monatlich verfügbare Haushaltsnettoeinkommen, also die Angaben über regelmäßige Einkünfte nach Abzug von Steuern und Sozialabgaben zuzüglich erhaltener Sozialtransfers. Die Monatseinkommen bilden die aktuell verfügbaren ökonomischen Ressourcen für alle zum Befragungszeitpunkt im Haushalt lebenden Personen ab. Um die Einkommenssituation von Haushalten unterschiedlicher Größe und Zusammensetzung vergleichbar zu machen, werden alle Haushaltseinkommen entsprechend dem inzwischen EU-weit standardisierten Vorgehen unter Verwendung der neuen (revidierten) OECD-Skala in sogenannte » ̈̈quivalenzeinkommen « umgerechnet. ${ }^{19}$ Alle Einkommensangaben werden in Euro ausgewiesen. Die Analysen erfolgen auf Personenebene und stellen eine statistische Repräsentation der in privaten Haushalten lebenden gesamten Bevölkerung in Deutschland. Die Anstaltsbevölkerung (etwa in Altersheimen) bleibt unberücksichtigt.

Zur Beurteilung der Kaufkraftentwicklung werden die Einkommen einheitlich zu Preisen des Jahres 2000 ausgewiesen. ${ }^{20}$ Die Preisanpassung erfolgt jährlich bezogen auf das Einkommensjahr. Schließlich werden die neu aufgenommenen Teilstichproben erst ab der jeweils zweiten Erhebungswelle berücksichtigt, um Schwankungen im zeitlichen Verlauf aufgrund methodischer Einflüsse im Antwortverhalten auszuschließen. Diese Einschränkung der befragten Fälle basiert auf Untersuchungen, die zeigen, dass auch repräsentative Längsschnittuntersuchungen ein in der ersten Welle abweichendes Antwortverhalten bei der Erfassung von Einkommen und anderen Indikatoren aufweisen. ${ }^{21}$

19 Die revidierte OECD-Skala weist der ersten Person im Haushalt ein Gewicht von $1 \mathrm{zu}$, jeder weiteren Person über 14 ein Gewicht von 0.5 und allen anderen ein Gewicht von 0.3. Zur näheren Besprechung von Äquivalenzskalen siehe beispielsweise Schwarze 2003.

20 Zugrunde gelegt wurden die offiziellen Preisindizes des Statistischen Bundesamts unter h ttp://www.destatis.de/jetspeed/portal/cms/Sites/destatis/Internet/DE/Content/Statistiken /Zeitreihen/LangeReihen/LebensunterhaltKonsum/Content100/lrleb02 a,templateId=re nderPrint.psml (Zugriff vom 12.07.2012).

21 Frick et al. 2006. Komplett ausgeschlossen wurde die Hocheinkommensstichprobe G, um Verzerrungen über die Zeit mit der Aufnahme einer solchen Stichprobe zu vermeiden. 
Das SOEP verfügt über detaillierte Informationen über die regionale Zugehörigkeit der befragten Haushalte. Die unten beschriebene Einteilung der Bundesrepublik Deutschland wurde auf der Ebene der Raumordnungsregionen errechnet und mit den Daten des SOEP verbunden. Als Beobachtungsperiode für die regionalen Analysen steht hier der Zeitraum 2000 bis 2006 zur Verfügung. Um bei den räumlichen Differenzierungen robuste Ergebnisse zu erzielen, wurden trotz der größeren Fallzahlen im SOEP ab 2000 je drei Jahre zu zwei Zeiträumen zusammengefasst: als Startperiode die Jahre 2000 bis 2002, als Endperiode die Jahre 2004 bis 2006. Zur Abschätzung der aktuellen Situation auf nationaler Ebene wurde allerdings die jährliche Betrachtungsweise beibehalten, wobei die Ergebnisse bis 2010 verwendet wurden.

\section{Methoden}

Bei der Messung der Einkommenspolarisierung gibt es bisher kein einheitliches Vorgehen. In empirischen Studien wird der Begriff der »Polarisierung « nicht immer eindeutig von »Ungleichheit " getrennt. Klassische Ungleichheitsindizes messen den Abstand zwischen den Einkommen einzelner Personen oder Gruppen einer Gesellschaft. Polarisierung hingegen richtet den Fokus nicht nur auf den Abstand zwischen den Einkommen, sondern auch auf die Anzahl der Personen mit niedrigen bzw. hohen Einkommen relativ zu den Personenanteilen im mittleren Einkommenssegment. Ungleichheit zeigt also die Abweichung vom allgemeinen Mittelwert einer Verteilung, wohingegen Polarisierung die Verdichtung um lokale Mittelwerte thematisiert. ${ }^{22}$ Polarisierung und wachsende Ungleichheit müssen daher nicht immer gleichzeitig auftreten, es kann sogar sein, das trotz steigender Polarisierung eine sinkende Ungleichheit zu beobachten ist, wenn sich die Spreizung der gesamten Einkommensverteilung zwar erhöht, sich die jeweiligen Gruppen an den Rändern der Verteilung aber ähnlicher werden. ${ }^{23}$

Eine so verstandene Messung von Polarisierung setzt - zumindest für eine deskriptive Darstellung - eine Einteilung der Einkommensverteilung in Gruppen voraus. Für diese Einteilung gibt es bisher keine allgemeine Vorgehensweise in der Literatur. Allerdings gibt es im Bereich der Armutsforschung Anhaltspunkte für eine mögliche Unterscheidung. Der am häufigsten verwendete Armutsbegriff in empirischen Studien beruht auf einem sogenannten relativen Einkommenskonzept und orientiert sich an der Definition der Europäischen Union. ${ }^{24}$ Gemäß den vom Statistischen Amt der EU (Eurostat) empfohlenen Schwellenwerten gilt demnach als einem erhöhten Armutsrisiko ausgesetzt, wer in einem Haushalt lebt, dessen Äquivalenzeinkommen weniger als 60 Prozent des Medians der Einkommen in der gesamten Bevölkerung beträgt. Ergänzend werden oftmals noch die Werte für »strenge Armut « (40 Prozent Median), "ausgeprägte Armut " (50 Prozent des Medians) und »Niedrigeinkom-

22 Amiel et al. 2010.

23 Eine ausführliche Darstellung dieses Phänomens findet sich in Esteban, Ray 1994.

24 Siehe Eurostat Task Force 1998.

Leviathan, 40. Jg., 3/2012 
men « (70 Prozent des Medians) dargestellt, zum Beispiel im Armuts- und Reichtumsbericht der Bundesregierung. ${ }^{25}$

Die Frage der Einkommenspolarisierung unterscheidet sich (wie oben bereits beschrieben) bei der Bewertung von Veränderungen der Einkommensverteilung gegenüber Einkommensungleichheit oder -armut allgemein dadurch, dass die beiden Ränder der Einkommensverteilung im Vergleich zur Mitte eine besondere Rolle spielen. ${ }^{26}$ Anders als bei der Armutsforschung interessieren dabei nicht nur Größe und Veränderung des unteren, sondern auch des oberen Randbereichs. Zur Messung der Polarisierung in der Einkommensverteilung werden daher meist drei Gruppen gebildet: Unten, Mitte und Oben. Eine allgemein anerkannte Praxis der empirischen Abgrenzung der oberen bzw. unteren Einkommensränder existiert allerdings nicht. Hier werden diese Einkommensbereiche daher in Anlehnung an die genutzten Definitionen im Armuts- und Reichtumsbericht der Bundesregierung gebildet. ${ }^{27}$

Der untere Einkommensbereich wird dort unter anderem als Niedrigeinkommensbereich beschrieben und bezieht sich auf Haushalte mit einem äquivalenzgewichteten Einkommen, das um mehr als 30 Prozent unter dem Medianeinkommen liegt. Der obere Einkommensbereich beginnt bei einem Haushaltseinkommen, das mindestens 50 Prozent über dem Medianwert liegt. Dies soll den Einkommensbereich mit gesichertem Wohlstand beschreiben. Damit ergeben sich folgende Gruppen: ${ }^{28}$

- Unterer Bereich (Niedrige Einkommen): Haushaltseinkommen bei weniger als 70 Prozent des Medians

- Mittlerer Bereich (Mittlere Einkommen): Haushaltseinkommen mindestens 70 Prozent des Medians, aber nicht mehr als 150 Prozent des Medianeinkommens

- Oberer Bereich (Hohe Einkommen): Haushaltseinkommen bei mehr als 150 Prozent des Medians

Eine mögliche Polarisierung in der Einkommensverteilung kann in unterschiedlichen Dimensionen untersucht werden. Eine Dimension wäre die Untersuchung der Personenanteile, die auf die drei Einkommensgruppen entfallen. Steigen im Zeitverlauf die Anteile der Ränder zu Lasten der Mitte, so liegt Einkommenspolarisierung vor (relative Polarisierung). Eine solche Betrachtung liegt beispielsweise der These vom Bedeutungsverlust der mittleren Einkommensgruppen zugrunde. ${ }^{29}$ Eine andere Dimension der Polarisierung ist die Distanz zwischen den Gruppen im Einkommensdurchschnitt. Nimmt beispielsweise der Abstand der jeweiligen gruppenspezifischen mittleren Einkommen zwischen den drei Gruppen zu, sprechen wir von einer verschärften Polarisierungstendenz (faktische Polarisierung). Eine dritte

25 Auch genutzt wird dies im europäisch vergleichbaren Satz der Laeken-Indikatoren.

26 Zur näheren Beschreibung des Konzepts der Einkommenspolarisierung vgl. Esteban, Ray 1994.

27 Bundesministerium für Arbeit und Soziales 2001, 2005, 2008.

28 Dies ist nicht deckungsgleich mit der Definition von Armut und Reichtum im Armutsund Reichtumsbericht, da diese jeweils etwas »schärfere « Grenzen nutzen (60 bzw. 200 Prozent des Medianeinkommens).

29 Grabka, Frick 2008; Goebel et al. 2010. 
Dimension der Polarisierung berücksichtigt auch die absoluten Einkommensveränderungen. Werden die Armen immer ärmer und die Reichen immer reicher, so drückt dies eine absolute Polarisierungstendenz aus.

Für die Abbildung der verschiedenen Dimensionen von Polarisierung der Einkommensverteilung bieten sich demnach zunächst die unmittelbare Analyse der Personenanteile der Einkommensgruppen und die Einkommensmittelwerte der jeweiligen Gruppen bzw. ihre Veränderungen an. Zur Beurteilung zeitlicher wie räumlicher Vergleiche ist die Verwendung einer einzigen Kennzahl (Index) mit klar definierten Grundannahmen (Axiome) zur Bewertung von Einkommenspolarisierung notwendig. Dies gilt umso mehr, wenn beide Dimensionen in der Bewertung zusammengeführt werden sollen. Werden bei der Analyse von Polarisierungstendenzen lediglich die Personenanteile in den definierten Gruppen beschrieben oder die sich verändernden Abstände der gruppenspezifischen Mittelwerte, dann ist es nicht möglich, zu entscheiden, welche der verglichenen Entwicklungen eine stärkere Polarisierung darstellt. Ist die Zunahme des Personenanteils von 5 Prozent in der untersten Gruppe ohne Verschiebung des Mittelwerts eine stärkere oder weniger starke Polarisierung als die in einer Situation, in der die unterste Einkommensgruppe zwar nur um 3 Prozent wächst, aber gleichzeitig Einkommensverluste hinnehmen muss?

Der Index, der der üblichen Beschreibung der Veränderung von Personenanteilen am nächsten kommt, ist der von Reynal-Querol ${ }^{30}$ vorgeschlagene Polarisierungsindex. Da die Autorin aus der politischen Konfliktforschung kommt, nutzt dieser Index nicht die zusätzliche Information des Einkommensabstands zwischen den Gruppen. Formal ist ihr Index definiert als

$$
P R Q(y)=1-\sum\left(\frac{0.5-\pi_{i}}{0.5}\right)^{2} \pi_{i}
$$

wobei $n$ die Anzahl der Gruppen darstellt und $\pi_{\mathrm{i}}$ die relative Größe der Gruppe $i$.

Nimmt man nur zwei Gruppen an, so ist der Index Null, wenn in einer der beiden Gruppen alle Personen liegen. Er nimmt seinen maximalen Wert an, wenn die Gruppen genau gleich groß sind (jeweils 50 Prozent der Bevölkerung; siehe Abbildung 1).

30 Reynal-Querol 2002.

Leviathan, 40. Jg., 3/2012 


\section{Abbildung 1: Verlauf des Reynal-Querol-Index bei zwei Gruppen}

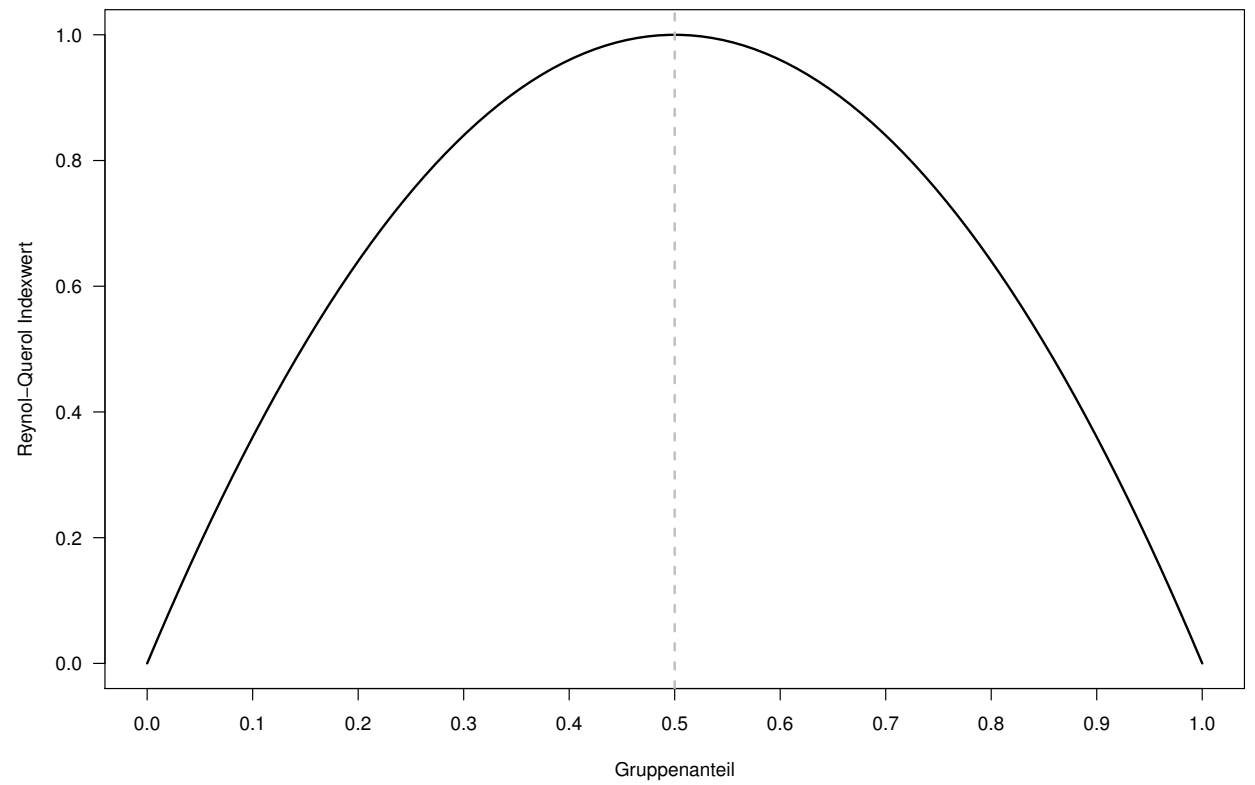

Quelle: eigene Darstellung.

Dieser Index kann somit Phänomene wie den Bedeutungsverlust der mittleren Einkommensgruppe (»shrinking middleclass «) mit dem Fokus auf Personenanteilen im Hinblick auf Polarisierung zusammenfassen. Der Nachteil ist dabei aber, dass die zusätzliche Information, die bei der Analyse von Einkommenspolarisierung vorliegt (der mittlere Einkommensabstand zwischen den Gruppen), nicht genutzt wird. ${ }^{31}$

Seit dem Erscheinen des Aufsatzes von Esteban und Ray 1994 gibt es Bestrebungen, die beiden Dimensionen der Polarisierung in einer einzigen Kennzahl (Index) zusammenzuführen. Grundlegend für die seither vorgeschlagenen Indizes ist das Bezugssystem von Identifikation und Entfremdung. Die dahinter liegende Idee ist relativ simpel: Polarisierung entsteht durch eine Entfremdung der unterschiedlichen (Einkommens-)Gruppen zueinander und eine gleichzeitige Identifikation der Personen innerhalb einer (Einkommens-)Gruppe. Allerdings ist damit noch nicht gesagt, wie die einzelnen Bestandteile zueinander gewichtet werden sollten.

Esteban und Ray formulierten nicht nur das »Identifikation und Entfremdungs «Bezugssystem, sondern schlugen auch noch eine empirische Umsetzung zu dessen Messung vor. ${ }^{32}$ Der von ihnen entwickelte Index gewichtet die Populations-Ver-

31 Montalvo und Reynal-Querol zeigten auf, das dieser Index im Prinzip das weiter unten beschriebene Maß von Esteban und Ray darstellt, allerdings umformuliert auf eine diskrete Verteilung und mit einem $\alpha$-Wert gleich eins; siehe Montalvo, Reynal-Querol 2005.

32 Esteban, Ray 1994. 
hältnisse der Gruppen zueinander mit dem absoluten Abstand zwischen den mittleren Einkommen in den jeweiligen Gruppen. Zur Abstandsmessung wird eine einfache euklidische Distanz benutzt. Der Index ist daher definiert als

$$
E R(\pi, y)=\sum_{i=1}^{n} \sum_{i=1}^{n} \pi_{i}^{1+\alpha} \pi\left|y_{i}-y_{j}\right|
$$

wobei $n$ auch hier die Anzahl der Gruppen darstellt, $\pi_{\mathrm{i}}$ die relative Größe der Gruppe $i$ und $y_{\mathrm{i}}$ das mittlere Einkommen in der Gruppe $i$. Der Parameter $\alpha$ bestimmt den Grad an Sensitivität des Maßes gegenüber Polarisierung, das heißt um wie viel stärker sich das Polarisierung-Maß von Standard-Ungleichheitsmaßen unterscheidet. Oder anders ausgedrückt: wie stark sich ein Individuum der Gruppe $i$ entfremdet fühlt gegenüber der Gruppe $j .{ }^{33}$ Ist der Parameter $\alpha$ gleich Null, stimmt dieses Polarisierungsmaß mit dem Gini-Koeffizienten zur Messung von Ungleichheit überein.

Esteban, Gradín und Ray schlugen zudem eine generalisierte Version dieses Index vor. ${ }^{34}$ In der generalisierten Fassung des Index wird ein »Fehlerterm « abgezogen, der aus der Differenz zwischen dem Gini der ursprünglichen und der gruppierten Einkommen entsteht. Dieser Fehlerterm, hervorgerufen durch die Gruppierung der Einkommensverteilung, wird minimiert, indem ein Algorithmus zur automatischen Festlegung der Gruppengrenzen genutzt wird. Der Forscher bestimmt nun nicht mehr die exakte Einkommensgrenze, sondern definiert nur die Anzahl der zu bildenden Gruppen. Die Gruppengrenzen werden dann automatisch so gewählt, dass die Streuung der Einkommen innerhalb der Gruppen minimiert wird oder, anders ausgedrückt, indem der später zur Korrektur abgezogene Fehlerterm minimiert wird. Die Diskussion zur Einkommenspolarisierung dreht sich häufig nur um die Veränderungen in den Personenanteilen der einzelnen Einkommensgruppen; die Wirkung der Einkommensverteilung innerhalb und zwischen diesen Gruppen wird meist ausgeblendet. Um die Komplementarität zu den deskriptiven Analysen der Anteilsverschiebungen zu gewährleisten, stellen wir in den Resultaten nur auf den von Esteban und Ray ursprünglich vorgeschlagenen Index ab, der die Grenzen zur Gruppierung der Einkommen festhält. Den weiterentwickelten Index nach Esteban, Gradín und Ray nutzen wir für Robustheitskontrollen.

Weitere in der Literatur vorgeschlagene Indizes zur Messung von Polarisierung sind unter anderem der Index von Wolfson ${ }^{35}$ oder der von Duclos, Esteban und Ray $^{36}$, die allerdings beide für die hier gewählte Fragestellung als nicht so geeignet erscheinen. Auf der einen Seite ist der Wolfson-Index (auch als Bi-PolarizationIndex bezeichnet), der die Einteilung von nur zwei Gruppen exakt am Median ausrichtet und somit die von uns gewählte Dreiteilung nicht widerspiegeln kann. Sozu-

33 Esteban und Ray zeigen weiterhin, das $\alpha$ nicht größer als 1,6 sein darf, da ansonsten die von ihnen definierten Axiome zur Messung von Polarisierung nicht erfüllt werden.

34 Esteban et al. 2007.

35 Wolfson 1994, 1997.

36 Duclos et al. 2004.

Leviathan, 40. Jg., 3/2012 
sagen das andere Extrem ist der von Duclos et al. vorgeschlagene Index, der vollständig auf eine Gruppeneinteilung verzichtet und über die Abschätzung einer Dichtefunktion die Polarisierung einer Verteilung beurteilt.

Zur Abschätzung der statistischen Fehlerwahrscheinlichkeit bei der Bestimmung der verwendeten Polarisationsmaße wurden die Konfidenzintervalle über ein Bootstrapping-Verfahren ermittelt. Wir haben dabei mit 500 Replikationen gearbeitet.

\section{Resultate}

\section{Entwicklung in Gesamtdeutschland}

Das Faktum einer zunehmenden Polarisierung der Einkommensverteilung ist seit Ende der 1970er Jahre für die USA nahezu unbestritten. ${ }^{37}$ Aber auch in Europa sind Phänomene zunehmender Einkommensdifferenzen seit längerem unübersehbar. ${ }^{38}$ Speziell in Deutschland hingegen wurde noch bis weit in die 1990er Jahre hinein davon ausgegangen, dass keine eindeutigen Trends in der Veränderung der Einkommensverteilung auszumachen sind. ${ }^{39}$

Von der Jahrtausendwende an nimmt aber die Einkommensdifferenzierung nicht nur in Europa, sondern auch in Deutschland spürbar zu. ${ }^{40}$ Die Ergebnisse weisen auf eine zumindest relative Polarisierung der Einkommensverteilung hin. Die Anteile der Haushalte in den mittleren Einkommensgruppen (sogenannte Mittelschicht) nehmen ab, diejenigen der oberen und unteren Gruppen dagegen tendenziell $\mathrm{zu}^{41}$

Betrachtet man die von uns verwendete Einteilung in drei Gruppen, fällt vor allem der Zuwachs des Anteils der Haushalte mit niedrigen Einkommen ins Auge (Abbildung 2). Dieser Trend setzte sich auch in den Jahren 2007 und 2008 fort, in denen die konjunkturelle Entwicklung deutlich aufwärts ging, ebenso wie im Krisenjahr 2009. Gleichzeitig ist der Anteil der Haushalte mit mehr als 150 Prozent des Medianeinkommens zwischen 2000 und 2008 nahezu kontinuierlich gestiegen. Der deutliche Rückgang des Anteils an Personen in Haushalten mit höherem Einkommen im Krisenjahr 2009 wurde bereits ein Jahr später wieder kompensiert.

37 Bernstein et al. 2002.

38 Anxo, Storrie 2000.

39 Hauser 2003,

40 Frick et al. 2005; Goebel, Krause 2007; OECD 2011.

41 Grabka, Frick 2008; Goebel et al. 2010. 
Abbildung 2: Entwicklung der Einkommenspolarisierung in Deutschland 1992 bis 2010: Anteile der Einkommensgruppen in Prozent

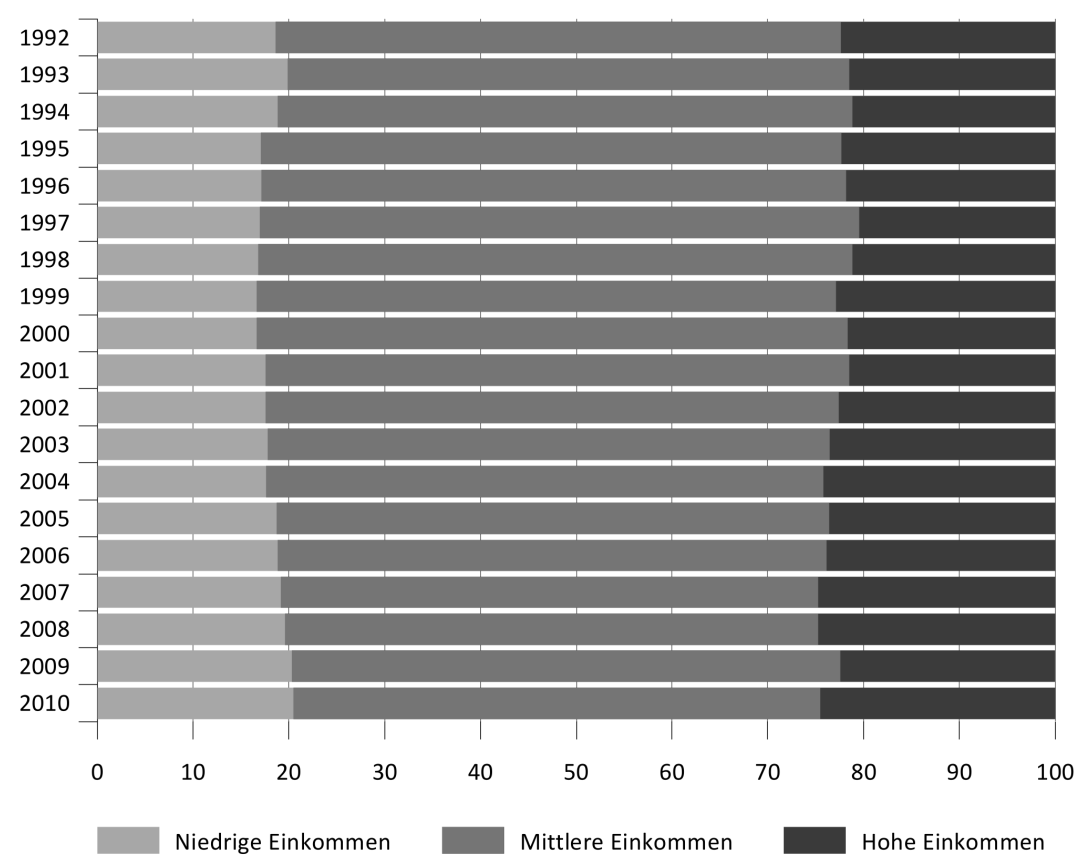

Quelle: SOEP.v 27, eigene Berechnungen.

Die für die regionale Analyse aufbereitete Beobachtungsperiode 2000 bis 2006 ist gekennzeichnet durch eine deutliche Zunahme der Personenanteile an den Rändern der Einkommensverteilung. Diese Anteilsverschiebung zeigt sich auch im Index von Reynal-Querol (Abbildung 3). Von 2000 bis 2008 nehmen die Anteile der Ränder der Einkommensverteilung durchgängig zu. Nach kurzer Unterbrechung liegt der Index auch 2010 über dem Wert von 2008. Der graue Bereich um die Linie für den Indexwert markiert den statistischen Unsicherheitsbereich bei einer Fehlerwahrscheinlichkeit von 5 Prozent. Im längerfristigen Vergleich sind demnach die Anteilsverschiebungen statistisch signifikant.

Einkommenspolarisierung meint aber nicht nur die bloßen Anteilsverschiebungen zwischen Einkommensgruppen. Absolute Einkommenspolarisierung liegt erst dann vor, wenn auch die Entwicklung der Einkommensmittelwerte der drei definierten Gruppen auseinandergeht. Beide Komponenten der Einkommenspolarisierung zeigen sich im Polarisationsindex nach Esteban-Ray. Dieser Polarisierungsindex steigt insbesondere zwischen 2000 und 2006 stark an (Abbildung 3). Die Polarisierung der Einkommen ist in Deutschland also gerade in der von uns für die regionalen Analysen ausgewählten Periode ausgeprägt; sie nimmt von 2000 bis 2006 um 15 
Prozent zu (Abbildung 3). In den Vor- bzw. Nachperioden weist der Esteban-RayIndex keine eindeutige statistisch signifikante Polarisierungstendenz aus.

Abbildung 3: Polarisationsindex in Deutschland 1992 bis 2010 nach Esteban-Ray (linke Achse) und Reynal-Querol (rechte Achse)

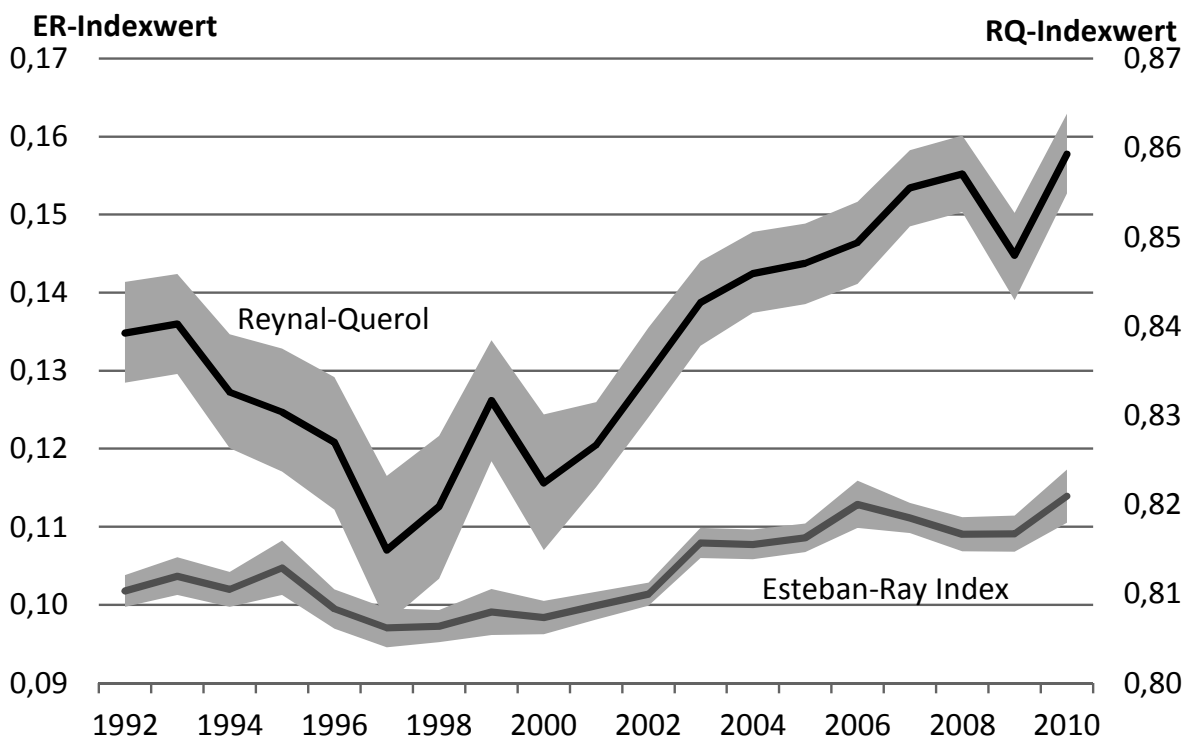

Quelle: SOEP.v27, eigene Berechnungen.

\section{Entwicklung in den Stadtregionen insgesamt}

Die Vermutung, dass die Einkommenspolarisierung vor allem ein Kennzeichen der Entwicklungen in den großen Städten sei, ist insbesondere von Saskia Sassen in ihrer Beschreibung der sogenannten »Global Cities « populär gemacht worden. ${ }^{42}$ Eine konkrete empirisch nachvollziehbare Definition dieses Regionstypus gibt es allerdings nicht. ${ }^{43}$ Wir analysieren zunächst alle großen Städte in Deutschland. Dabei werden allerdings nicht nur die Kernstädte, sondern die Ballungsräume insgesamt einbezogen, um die relevanten räumlichen Wirtschaftseinheiten zu erfassen. Die

42 Sassen 1994.

43 Saskia Sassen selbst vermutet Strukturen einer »Global City « in Deutschland am ehesten im Rhein-Main-Gebiet und in Berlin, ohne dabei jedoch nachvollziehbare quantitative Abgrenzungskriterien zu verwenden; vgl. Sassen 2001. 
konkrete Abgrenzung erfolgt entsprechend der Analyseregionen auf der Basis der Raumordnungsregionen des Bundesamtes für Bauwesen und Raumordnung. ${ }^{44}$

Tabelle 2 weist für die Stadtregionen und die übrige Bundesrepublik die Haushaltsanteile und Mittelwerte für die drei Einkommensgruppen bezogen auf die beiden Periodendurchschnitte 2000 bis 2002 und 2004 bis 2006 aus. Die Tendenz zum Bedeutungsverlust der mittleren Einkommensgruppe (relative Einkommenspolarisierung) scheint dabei kein besonderes Phänomen der großen Städte zu sein. Die Anteile der Haushalte mit hohen und niedrigen Einkommen steigen und die der mittleren fallen, dies gilt sowohl im Durchschnitt der Stadtregionen als auch im Mittel der übrigen Regionen. Die Anteilsverschiebungen sind allerdings bei den großen Städten stärker. Insbesondere der Anteil der unteren Einkommensgruppe nimmt deutlicher zu. Der Reynal-Querol-Index - als Maß für die relative Einkommenspolarisierung - nimmt in beiden Regionsgruppen statistisch signifikant zu. Im Durchschnitt ist der Zuwachs in den Stadtregionen größer.

Bei den Stadtregionen insgesamt werden zudem nicht nur die Ränder der Einkommensverteilung mengenmäßig immer größer, sondern es wurden auch »die Reichen reicher « und »die Armen ärmer «. Der Esteban-Ray-Index, der Anteils- wie Mittelwertveränderungen berücksichtigt, weist eine statistisch signifikante Zunahme der Polarisierung aus. Im Unterschied zum Typus der absoluten Einkommenspolarisierung verlieren die mittleren Einkommen aber in den Stadtregionen doppelt. Nicht nur ihre Haushaltsanteile gehen zurück, sondern auch der Einkommensdurchschnitt nimmt überdurchschnittlich stark ab.

Außerhalb der Stadtregionen beschränkt sich die Polarisierung auf einen anteilsmäßigen Rückgang der mittleren Einkommen. Ihr Einkommensdurchschnitt sinkt hingegen ähnlich wie bei der oberen Einkommensgruppe. Die Erhöhung der Einkommenspolarisierung, bestimmt mit dem Esteban-Ray-Index, ist im Mittelwert um gut 40 Prozent geringer als in den Stadtregionen. Die Polarisierungstendenz in den Nicht-Agglomerationen ist zudem bei einer Fehlerwahrscheinlichkeit von 5 Prozent statistisch nicht signifikant.

44 Siehe BBSR, http://www.bbsr.bund.de/cln_032/nn_1067638/BBSR/DE/Raumbeobachtu ng/Raumabgrenzungen/Raumordnungsregionen/raumordnungsregionen__node.html?_ _nnn=true (Zugriff vom 18.07.2012). 
Tabelle 2: Polarisierung nach Agglomerationsraum und in den untersuchten Perioden 2000-2002 und 2004-2006

\begin{tabular}{|c|c|c|c|c|c|c|}
\hline & \multicolumn{3}{|c|}{ Agglomerationsraum } & \multicolumn{2}{|c|}{ Kein Agglomerationsraum } & \multirow[b]{2}{*}{ Änderung } \\
\hline & 2000-2002 & 2004-2006 & Änderung & $2000-2002$ & 2004-2006 & \\
\hline \multicolumn{6}{|c|}{ Anteil von Personen in Haushalten mit ... Einkommen } & \\
\hline Niedrigem & $17,5 \%$ & $20,3 \%$ & $16,5 \%$ & $21,1 \%$ & $23,0 \%$ & $8,9 \%$ \\
\hline Mittlerem & $62,8 \%$ & $58,1 \%$ & $-7,6 \%$ & $64,4 \%$ & $61,3 \%$ & $-4,8 \%$ \\
\hline Hohem & $19,7 \%$ & $21,6 \%$ & $9,5 \%$ & $14,4 \%$ & $15,6 \%$ & $8,2 \%$ \\
\hline \multirow{4}{*}{$\begin{array}{l}\text { Polarisationsinc } \\
\text { Reynal-Querol } \\
\text { 95\%-Konfi- } \\
\text { denzband }\end{array}$} & 0.812 & 0.844 & $3,9 \%$ & 0.803 & 0.828 & $3,1 \%$ \\
\hline & {$[, 806-, 819]$} & {$[, 839-, 849]$} & {$[2,4-5,3]$} & {$[, 796-, 810]$} & {$[, 821-, 834]$} & {$[1,3-4,7]$} \\
\hline & \multicolumn{3}{|c|}{ Agglomerationsraum } & \multicolumn{2}{|c|}{ Kein Agglomerationsraum } & \\
\hline & $2000-2002$ & 2004-2006 & Änderung & $2000-2002$ & 2004-2006 & Änderung \\
\hline \multicolumn{6}{|c|}{ Mittleres Einkommen $(€)$ von Personen in Haushalten mit ... Einkommen } & \\
\hline Niedrigem & 632 & 628 & $-0,6 \%$ & 634 & 615 & $-3,0 \%$ \\
\hline Mittlerem & 1.234 & 1.211 & $-1,9 \%$ & 1.197 & 1.187 & $-0,8 \%$ \\
\hline Hohem & 2.388 & 2.433 & $1,9 \%$ & 2.376 & 2.352 & $-1,0 \%$ \\
\hline Insgesamt & 1.356 & 1.356 & $0,0 \%$ & 1.248 & 1.237 & $-0,9 \%$ \\
\hline \multirow{2}{*}{$\begin{array}{l}\text { Polarisationsinc } \\
\text { Esteban-Ray } \\
95 \%-K o n f i- \\
\text { denzband }\end{array}$} & 0.112 & 0.118 & $5,3 \%$ & 0.109 & 0.112 & $3,3 \%$ \\
\hline & {$[, 110-, 114]$} & {$[, 116-, 120]$} & {$[2,3-8,3]$} & {$[, 107-, 111]$} & {$[, 109-, 115]$} & {$[-1,3-7,9]$} \\
\hline
\end{tabular}

Quelle: SOEP.v 26, eigene Berechnungen.

\section{Entwicklung in unterschiedlichen Stadtregionstypen}

Ein wesentlicher Impuls für die Polarisierung der Haushaltseinkommen geht von der Veränderung der wirtschaftlichen Wachstumskräfte aus. Die Tertiarisierung - so die These - schafft die Potenziale für eine höhere Lohnspreizung und damit die Polarisierung der Haushaltseinkommen. ${ }^{45}$ Zur empirischen Überprüfung der These, dass die Intensität der Polarisierung von der industriellen bzw. tertiären Ausrichtung der Wachstumskräfte abhängt, ist eine Klassifizierung der Regionen nach dem Entwicklungsniveau (Wachstum vs. Schrumpfung) und der Art der Veränderung (Industrie vs. Dienstleistungen) erforderlich. Als Leitindikator für die Entwicklungsmuster betrachten wir die Veränderungen der Beschäftigung nach Verflechtungsregionen. Dazu nutzen wir die Entwicklung der Zahl der sozialversicherungspflichtig Beschäftigten 1998 bis 2006 der großstädtischen Analyseregionen.

45 Harrison, Bluestone 1988. 
Kontrovers diskutiert wird in der empirischen Forschung allerdings die Abgrenzung zwischen Dienstleitungen und Industrie. ${ }^{46}$ So werden auf der einen Seite auch in den Industrieunternehmen Dienstleistungstätigkeiten ausgeführt (Dienstleistungsgehalt der Warenproduktion); auf der anderen Seite wird ein bestimmter Teil der Vorleistungen für die Produktion von Dienstleistungsunternehmen direkt für die Industrie erstellt. Da es hier um den prägnanten Einfluss auf den regionalen Entwicklungsprozess geht, wird eine vereinfachende Methode angewandt. Wir betrachten jeweils den Sektor als Ganzes und tolerieren entsprechende Überschneidungen. Der Industriesektor wird durch das produzierende Gewerbe und produktionsbezogene Dienstleistungen (Großhandel, Logistik, Unternehmensdienste) abgebildet, der Dienstleistungssektor durch alle Dienstleistungsbereiche und die Dienstleistungstätigkeiten im produzierenden Gewerbe.

Aus der Gegenüberstellung der Entwicklungstendenzen der Beschäftigung in beiden Sektorbereichen und der Veränderung in der Gesamtbeschäftigung lassen sich vier Entwicklungstypen unterscheiden: ${ }^{47}$

- Generelles Wachstum (Zunahme Industrie; Zunahme Dienstleistungen)

- Tertiäres Wachstum (Abnahme Industrie; Zunahme Dienstleistungen; positiver Gesamteffekt)

- Industrielle Schrumpfung (Abnahme Industrie; Zunahme Dienstleistungen; negativer Gesamteffekt)

- Generelle Schrumpfung (Abnahme Industrie; Abnahme Dienstleistungen)

Die Ergebnisse der Typisierung nach der Entwicklungsdynamik sind in der Abbildung 4 dargestellt. Der Typus Generelle Schrumpfung findet sich danach in den meisten Stadtregionen Ostdeutschlands, Generelles Wachstum ist vor allem in den südlichen Agglomerationsräumen anzutreffen. Der Typus Industrielle Schrumpfung dominiert die Entwicklung in den Stadtregionen Nordrhein-Westfalens und Niedersachsens.

46 Kalmbach et al. 2005; Grömling et al. 1998.

47 Zwei weitere mögliche Typen, die sich bei der Kombination von Wachstum Industrie und Schrumpfung Dienstleistungen (einmal mit positivem, einmal mit negativem Gesamteffekt) ergeben könnten, kommen im Datensatz nicht vor. 


\section{Abbildung 4: Stadtregionen nach Entwicklungstyp}
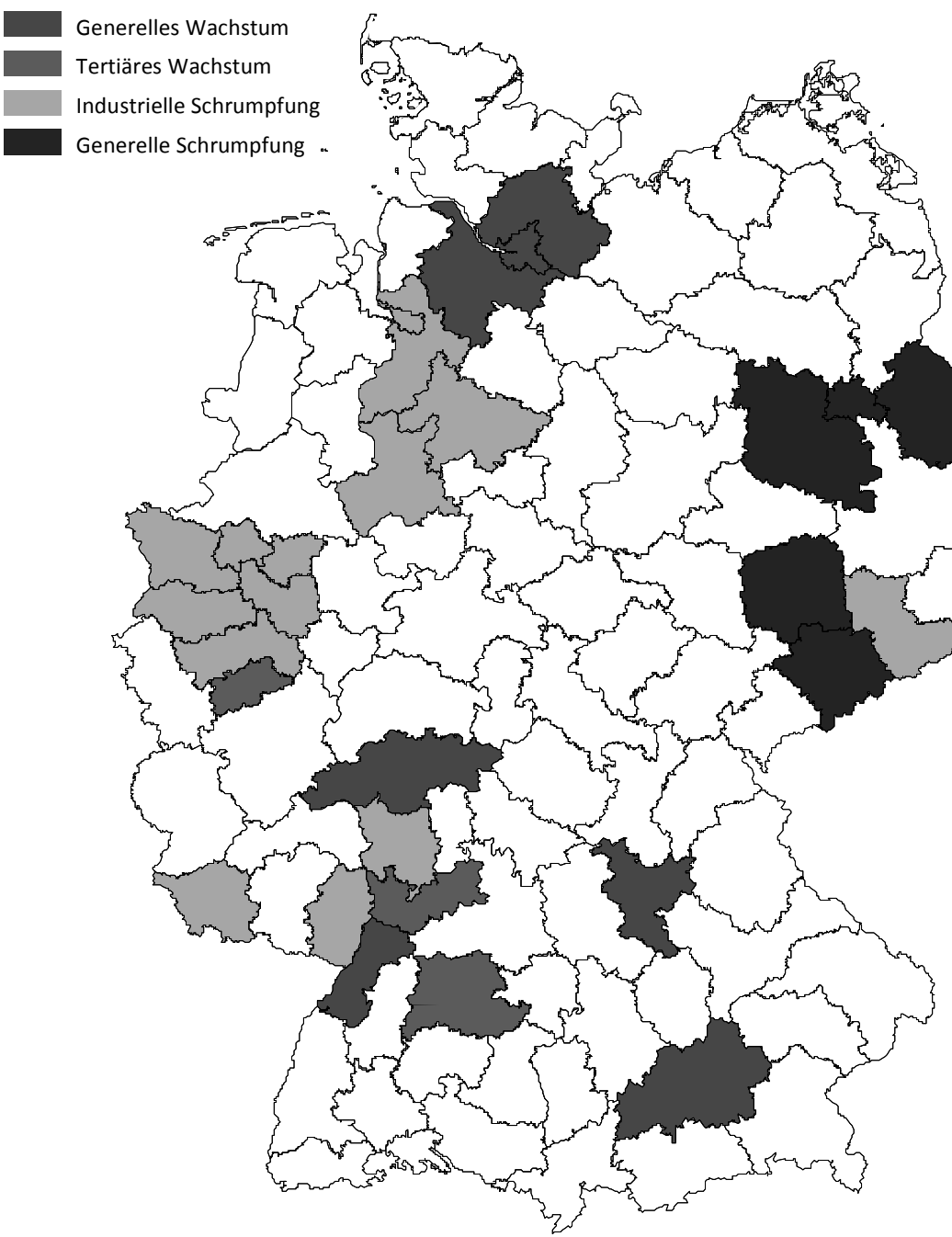

Die Auswertungen der Anteilsveränderung der Einkommensgruppen weisen für alle Entwicklungstypen einen Verlust bei den Haushalten mit mittleren Einkommen aus (Abbildung 5 oben). Das Phänomen der schrumpfenden mittleren Einkommensgruppe scheint somit ein generelles Entwicklungsmuster in den Stadtregionen bzw. in Deutschland zu sein, das weitgehend unabhängig vom wirtschaftlichen Entwicklungstyp der Stadtregionen ist. Es ist aber nicht in jedem Fall auch mit einer eindeutigen relativen Einkommenspolarisierung verbunden. So nimmt beim Entwicklungstyp Generelle Schrumpfung nicht nur der Anteil von Haushalten mit mitt- 
lerem, sondern auch der von Haushalten mit hohen Einkommen ab. In den Stadtregionen mit Generellem Wachstum wiederum verändert sich der Anteil der Haushalte mit hohem Einkommen kaum.

Abbildung 5: Relative Veränderung der Haushaltsanteile und der mittleren Einkommen nach Einkommensgruppen - Vergleich der Periode 2004-2006 zu 2000-2002, Angaben in Prozent

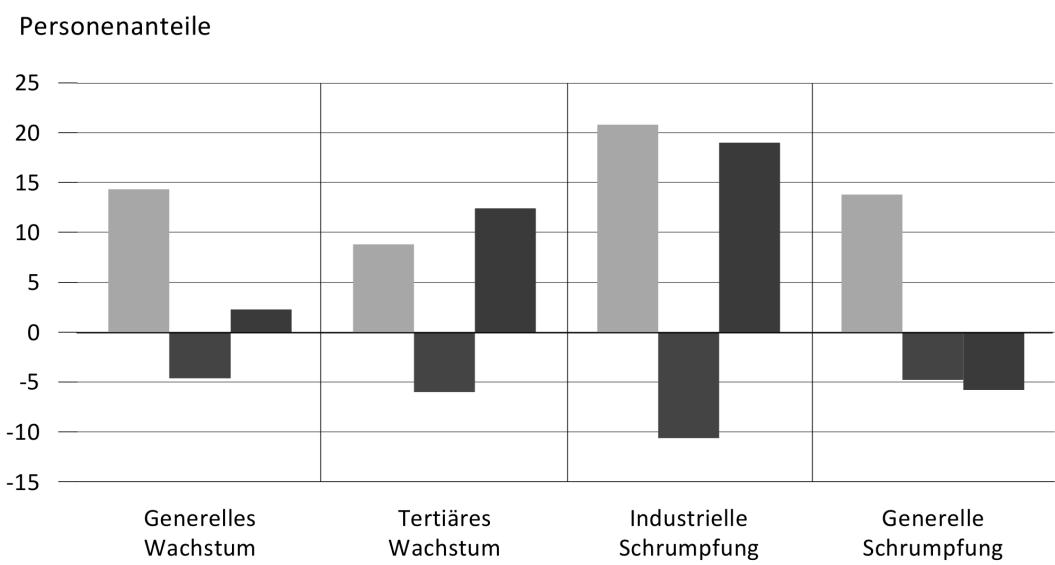

Einkommensmittelwerte

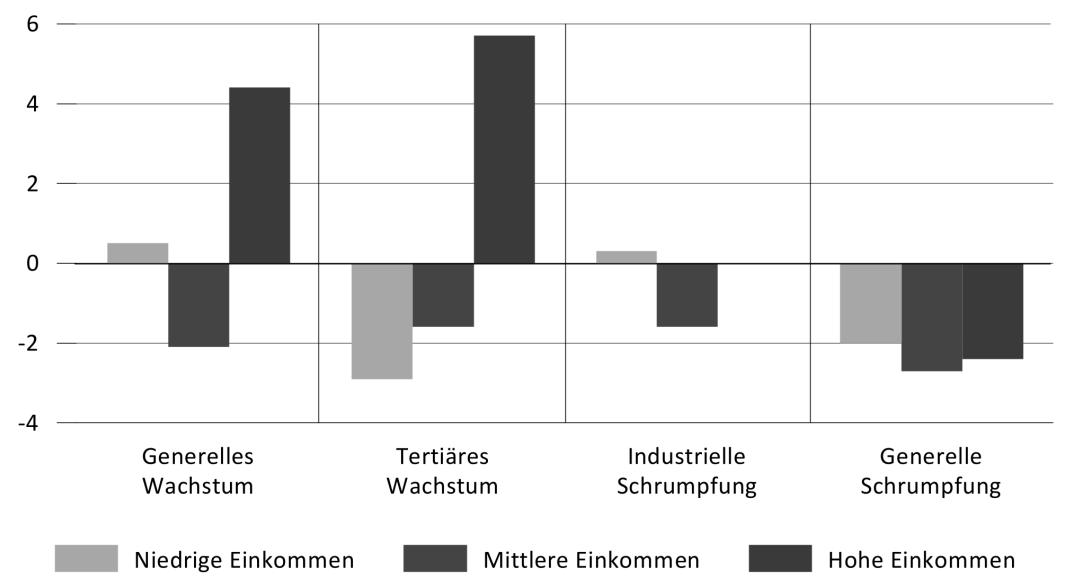

Quelle: SOEP.v 26, eigene Berechnungen.

Bei den Stadtregionen mit ausgeprägten wirtschaftlichen Strukturverschiebungen zwischen Industrie und Dienstleistungen dagegen deuten die Anteilsverschiebungen zwischen den Haushaltsgruppen im Vergleich der Perioden 2000-2002 und 2004-2006 auf eine starke relative Einkommenspolarisierung hin. Beim Ent- 
wicklungstyp Tertiäres Wachstum wächst der Anteil der unteren Einkommensgruppe um 9 Prozent, der der oberen um 12 Prozent. Noch stärker ist die Intensität der Einkommenspolarisierung in Stadtregionen vom Typ Industrielle Schrumpfung. In diesen Stadtregionen wachsen die Haushaltsanteile der Ränder um jeweils rund 20 Prozent.

Die Ergebnisse zu den Entwicklungen der Mittelwerte der Einkommensgruppen zueinander weichen von der reinen Anteilsverschiebung teilweise deutlich ab (Abbildung 5 unten). Besonders stark auseinander laufen die Entwicklungstrends im Regionstyp Tertiäres Wachstum. Das durchschnittliche Einkommen der unteren Einkommensgruppe geht stark zurück (-3 Prozent), das der oberen Einkommensgruppe dagegen nimmt stark zu (+6 Prozent). Hier werden die Ärmeren und Reicheren also nicht nur mengenmäßig mehr, sondern sie entfernen sich auch deutlich vom Einkommensdurchschnitt der Mittelgruppe (absolute Einkommenspolarisierung). Mit 4 Prozent finden wir hohe Einkommenszuwächse der Reicheren auch im Regionstyp Generelles Wachstum. Anders als bei den Regionen mit Tertiärem Wachstum erweisen sich die durchschnittlichen Einkommen der unteren Einkommensgruppe aber als stabil, während die der mittleren Einkommensgruppe deutlich zurückgehen. Eine absolute Einkommenspolarisierung liegt also beim Typ Generelles Wachstum nicht vor.

Wesentlich geringer sind die Unterschiede in den Veränderungsraten der durchschnittlichen Einkommensgruppenmittel in den Regionen mit industrieller Schrumpfung und genereller Schrumpfung. Beim Typus Industrielle Schrumpfungsregion dürfte dies vor allem Folge der Auflösungstendenzen der mittleren Einkommensgruppe sein. Viele Haushalte, die 2000-2002 noch zur mittleren Gruppe zählten, dürften nun in den unteren bzw. oberen Einkommensgruppen zu finden sein. Sie haben dort offensichtlich die durchschnittliche Einkommensentwicklung im unteren Segment stabilisiert und im oberen gebremst. Die Entwicklung im Typus Generelle Schrumpfungsregion ist auch bei den Einkommensmittelwerten durch eine Abstiegs- und nicht Polarisierungstendenz geprägt. In allen drei Einkommensgruppen gehen die Einkommenswerte im Durchschnitt zurück.

Eine Beantwortung der Frage, in welchen Regionstypen die Einkommenspolarisierung in Deutschland besonders ausgeprägt ist, lässt sich angesichts der teils gegenläufigen Ergebnisse nur aus der Zusammenführung von Anteils- und Mittelwertentwicklung beantworten. Eine solche Zusammenführung bietet insbesondere der Polarisierungsindex nach Esteban-Ray. Führt man die Analysen zur Veränderung der Haushaltsanteile und der Verschiebung der Einkommensmittelwerte zusammen, weist der Esteban-Ray-Index im Mittel in allen vier Regionstypen eine Zunahme der Einkommenspolarisierung im Vergleich der Perioden 2004-2006 zu 2000-2002 aus (Abbildung 6). 
Abbildung 6: Polarisationsindex nach Esteban-Ray zur Einkommensentwicklung in Agglomerationsräumen der Perioden 2000-2002 und 2004-2006.

Veränderung des Index in Prozent

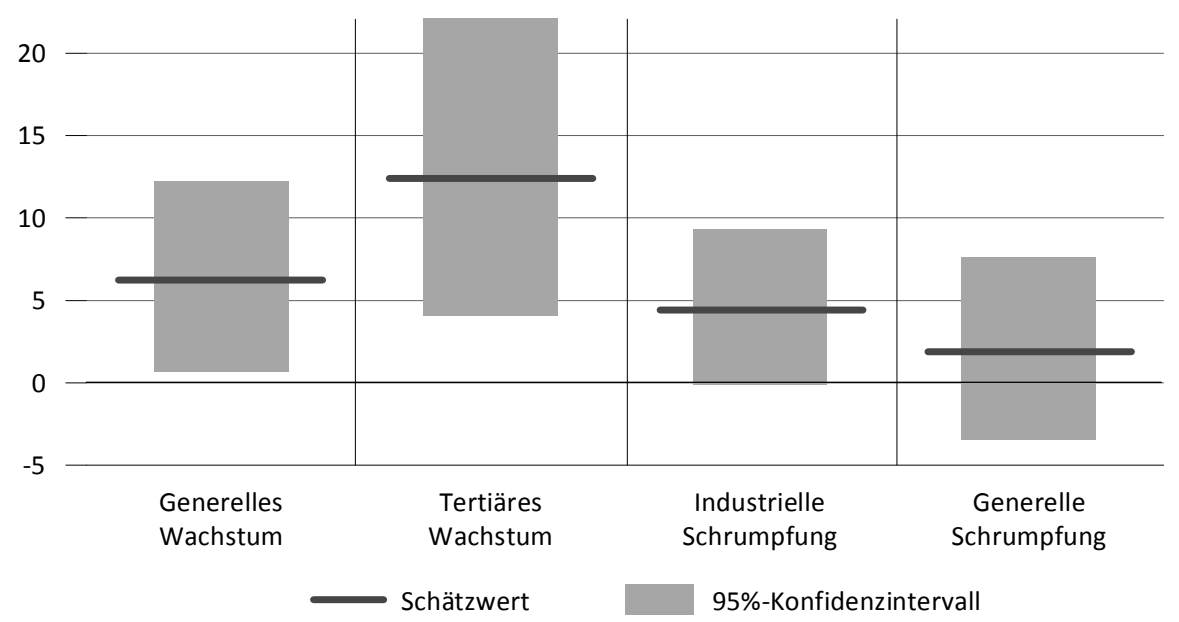

Quelle: SOEP.v 26, eigene Berechnungen.

In der Kombination von Anteils- und Mittelwertveränderungen ist die stärkste Zunahme der Einkommensgegensätze im Regionstyp Tertiäres Wachstum zu beobachten. ${ }^{48}$ Der Polarisationsindex nahm im Vergleich der beiden Perioden um fast 13 Prozent zu. Das Ausmaß der Einkommenspolarisierung weist allerdings eine hohe statistische Unsicherheit auf (grau markierter Bereich). Bei einer Irrtumswahrscheinlichkeit von 5 Prozent liegt der obere Wert für die Zunahme der Einkommenspolarisierung bei 22 Prozent, der untere Wert bei 4 Prozent.

Die Schätzwerte für die Einkommenspolarisierung in den Regionstypen Generelles Wachstum und Industrielle Schrumpfung liegen deutlich unter dem für den Regionstyp Tertiäres Wachstum. Der Polarisierungsindex nahm im Typ Generelles Wachstum um 6 Prozent, im Typ Industrielle Schrumpfung um 4 Prozent zu. Die Polarisierungstendenz ist dabei in beiden Fällen auf dem 95-Prozent-Niveau nur knapp statistisch signifikant. ${ }^{49}$

Die Entwicklungen in den Stadtregionen des Typs Generelle Schrumpfung sind dagegen erwartungsgemäß auch bei der Zusammenführung von Anteils- und Mittelwertveränderungen nicht als Einkommenspolarisierung interpretierbar. Zwar steigt der Polarisierungsindex im Vergleich der beiden Perioden auch hier an. Der

48 Dieses Ergebnis bestätigt sich auch bei Verwendung des EGR-Index (siehe Anhang, Tabelle A2).

49 Die Untergrenze der Bootstrap-Verteilung des Polarisationsindex 2004-2006 liegt nahe bei der Obergrenze der Periode 2000-2002 (unterer grau markierter Bereich). 
Zuwachs aber ist mit weniger als 2 Prozent vergleichsweise gering und der Unterschied auf dem 95-Prozent-Niveau zudem nicht statistisch signifikant.

\section{Fazit}

Das Phänomen des Bedeutungsverlusts der mittleren Einkommensgruppen zählt mittlerweile auch in Deutschland zu den prägnantesten empirischen Merkmalen des sozialstrukturellen Wandels. Die Anteilsverluste der mittleren Einkommensgruppen sind dabei zeitlich wie räumlich durchgehend feststellbar. Zwar bestehen im Konjunkturverlauf und im regionalen Vergleich Unterschiede in der Intensität, das Phänomen selbst ist aber überall und zu jeder Zeit zu beobachten.

Die immer wieder im Zusammenhang mit dem Rückgang von mittleren Einkommensbeziehern genannte zunehmende Einkommenspolarisierung ist dagegen im zeitlichen Trend sehr viel weniger stabil, vor allem aber zeigen sich große regionale Differenzen. So ist in Deutschland Einkommenspolarisierung in erster Linie ein Phänomen der großen Stadtregionen. Aber auch zwischen Stadtregionen mit nach Niveau und Art verschiedener wirtschaftlicher Dynamik zeigen sich markante Unterschiede.

Besonders deutlich nimmt die Einkommenspolarisierung in Stadtregionen zu, die durch einen starken Strukturwandel von der Industrie zu den Dienstleistungen geprägt sind. Dort, wo Deindustrialisierung und dynamisches Wachstum neuer Dienstleistungen aufeinandertreffen, werden die ärmeren Haushalte mehr und ärmer und die reicheren Haushalte reicher und mehr. Ein solches tertiäres Wachstum finden wir in Stadtregionen wie Stuttgart oder Mannheim. Aber auch dort, wo das Wachstum der Dienstleistungen nicht ausreicht, um die Verluste der Industrie auszugleichen, wie an Rhein und Ruhr, sind Polarisierungstendenzen erkennbar. Insbesondere die ehemals starke mittlere Einkommensgruppe zeigt hier einen mittlerweile auch längerfristig stabilen Abwärtstrend. Unsere empirische Arbeit für Deutschland bestätigt also vor allem die Überlegungen zum Einfluss der Deindustrialisierung auf die Einkommensverteilung, wie sie Mitte der 1980er Jahre für die Städte in den USA formuliert wurden.

Die Metropolen in Deutschland mit einem breiten dynamischen Wirtschaftswachstum, etwa die Stadtregionen von Frankfurt, München oder Hamburg, sind hingegen nicht die Spitzenreiter der Einkommenspolarisierung. Zwar nimmt im Durchschnitt der Polarisationsindex auch hier zu, aber beispielsweise die Einkommenswerte der unteren Einkommensgruppen erweisen sich hier als sehr stabil. Ein Grund hierfür dürfte sein, dass die genannten Regionen sich nicht nur im Dienstleistungssektor, sondern auch im Industriesektor positiv entwickelt haben.

In Berlin und den anderen größeren Stadtregionen Ostdeutschlands zeigt sich, dass Stagnation und Schrumpfung offensichtlich auch nicht mehr Einkommensgleichheit schaffen. Zwar zeigen sich unter den Bedingungen von abnehmender Beschäftigung in der Industrie wie im Dienstleistungssektor keine ausgeprägten Polarisierungstendenzen, wohl aber eine Abwärtsspirale, bei der nur die unteren Einkommensgrup- 
pen größer werden und alle anderen Haushaltsgruppen Einkommensverluste hinnehmen müssen.

Für die Stadtpolitik ist das vorgelegte Ergebnis in mehrfacher Hinsicht relevant. Aus der Sicht der Gesamtstadt heißt dies beispielsweise, dass durch die Stabilisierung der Industrie in den großen Städten nicht nur generelle Wachstumsimpulse erhalten und erschlossen werden, sondern auch, dass damit indirekt ein wichtiger Beitrag zur sozialen Kohäsion in den Städten geleistet wird. Der zunehmenden Heterogenität der Stadtentwicklung wird in Zukunft Rechnung getragen werden müssen, wird sie doch nicht nur in der allgemeinen gesellschaftlichen Dynamik, sondern - wie gezeigt - insbesondere auch in den sozialstrukturellen Polarisierungen spürbar. Hier gilt es, durch weitergehende, beispielsweise typspezifische Mobilitätsanalysen des Auf- und Abstiegs zwischen den Einkommensgruppen besondere sozialstrukturelle Problemlagen besser als bisher zu erfassen und damit die Chancen für Maßnahmen sozialer Kohäsion zu verbessern.

\section{Literatur}

Ähnelt, Reinhard; Goebel, Jan; Gornig, Martin; Häußermann, Hartmut 2009. »Soziale Ungleichheit und sozialräumliche Strukturen in deutschen Städten ", in Informationen zur Raumentwicklung 6, S. 405-414.

Amiel, Yoram; Cowell, Frank; Ramos, Xavier 2010. "Poles apart? An analysis of the meaning of polarization ", in Review of Income and Wealth 56, 1, S. 23-46.

Anxo, Dominique; Storrie, Donald 2000. The job creation potential of the service sector in Europe. Luxemburg: European Commission.

Bade, Franz-Josef 2006. Gewinner und Verlierer der wissensbasierten Ökonomie. Tagung "Wirtschaftsförderung in der Wissensgesellschaft « der Evangelischen Akademie Loccum.

Baumol, William J. 1967. » Macroeconomics of unbalanced growth: the anatomy of urban crisis ", in American Economic Review 57, S. 416-426.

Bernstein, Jared; McNichol, Elizabeth; Nicholas, Andrew 2002. Pulling apart: a state-by-state analysis of income trends. Report. Washington D.C.: Center on Budget and Policy Priorities and Economic Policy Institute.

Blau, Peter M. 1980. "Implications of growth in services for social structure ", in Social Science Quarterly 61, S. 3-22.

Bundesministerium für Arbeit und Soziales 2001. Lebenslagen in Deutschland. Der erste Armutsund Reichtumsbericht der Bundesregierung. Bonn.

Bundesministerium für Arbeit und Soziales 2005. Lebenslagen in Deutschland. Der 2. Armutsund Reichtumsbericht der Bundesregierung. Berlin.

Bundesministerium für Arbeit und Soziales 2008. Lebenslagen in Deutschland. Der 3. Armutsund Reichtumsbericht der Bundesregierung. Berlin.

Burger, Jack; Musterd, Sako 2002. »Understanding urban inequality: a model based on existing theories and an empirical illustration «, in International Journal of Urban and Regional Research 26, S. 403-413.

DIW (Deutsches Institut für Wirtschaftsforschung Berlin) 2008. »25 Wellen Sozio-oekonomisches Panel ", in Vierteljahrshefte zur Wirtschaftsforschung/Quarterly Journal of Economic Research 77, 3, S. 9-14.

Duclos, Jean-Yves; Esteban, Joan M.; Ray, Debraj 2004. »Polarization: concepts, measurement, estimation ", in Econometrica 72, 6, S. 1737-1772.

Esteban, Joan M.; Gradín, Carlos; Ray, Debraj 2007. »An extension of a measure of polarization, with an application to the income distribution of five OECD countries ", in Journal of Economic Inequality 5, 1, S. 1-19.

Esteban, Joan M.; Ray, Debraj 1994. »On the measurement of polarization ", in Econometrica 62, 4, S. 819-851.

Leviathan, 40. Jg., 3/2012 
Eurostat Task Force 1998. Recommendation on social exclusion and poverty statistics, document CPS/98/31/2. Luxembourg: Statistical Office of the European Communities.

Friedrichs, Jürgen; Häußermann, Hartmut; Siebel, Walter. Hrsg. 1986. Süd-Nord-Gefälle in der Bundesrepublik. Opladen: Leske + Budrich.

Frick, Joachim R. et al. 2005. "Zur langfristigen Entwicklung von Einkommen und Armut in Deutschland «, in Wochenbericht des DIW 4, S. 59-68.

Frick, Joachim R. et al. 2006. "Using analysis of gini (ANOGI) for detecting whether two subsamples represent the same universe: the German Socio-Economic Panel Study (SOEP) experience ", in Sociological Methods \& Research 34, 4, S. 427-468.

Gatzweiler, Hans-Peter; Milbert, Antonia; Sturm, Gabriele 2009. »Potenziale deutscher Städte in Zeiten des Wandels. Analysen auf Basis der Raum- und Stadtbeobachtung des BBSR «, in Informationen zur Raumentwicklung 3-4, S. 157-180.

Geppert, Kurt; Gornig, Martin 2006. »Renaissance der großen Ballungsräume in Deutschland: Wiedererstarkung im Westen, noch mangelnde Dynamik im Osten «, in Informationen zur Raumentwicklung 9, S. 505-513.

Goebel, Jan et al. 2008. »Mikrodaten, Gewichtung und Datenstruktur der Längsschnittstudie Sozio-oekonomisches Panel (SOEP) «, in DIW Vierteljahreshefte zur Wirtschaftsforschung 77, 3, S. 77-109.

Goebel, Jan; Gornig, Martin; Häußermann, Hartmut 2010. »Polarisierung der Einkommen: Die Mittelschicht verliert ", in Wochenbericht des DIW 24, S. 2-8.

Goebel, Jan; Habich, Roland; Krause, Peter 2006. »Einkommen - Verteilung, Angleichung, Armut und Dynamik ", in Datenreport 2006. Zablen und Fakten über die Bundesrepublik Deutschland, hrsg. v. Statistisches Bundesamt, S. 607-624. Bonn: Bundeszentrale für politische Bildung.

Goebel, Jan; Krause, Peter 2007. »Gestiegene Einkommensungleichheit in Deutschland «, in Wirtschaftsdienst 87, 12, S. 824-832.

Gornig, Martin 2005. »Polarisierung der wirtschaftlichen Potenziale - Folgen von Tertiärisierung und Europäisierung für die Städte ", in Deutsche Zeitschrift für Kommunalwissenschaften 44, II. Halbjahresband, S. 50-63.

Gornig, Martin; Häußermann, Hartmut 1998. "Städte und Regionen im Süd/Nord- und West/ Ost-Gefälle", in Kommunalpolitik, hrsg. v. Wollmann, Hellmut; Roth, Roland, S. 338-360. Bonn: Bundeszentrale für politische Bildung.

Grabka, Markus M. 2007. Codebook for the \$PEQUIV file 1984-2006. Data documentation, Nr. 21. Berlin: DIW.

Grabka, Markus M.; Frick, Joachim R. 2008. „Schrumpfende Mittelschicht: Anzeichen einer dauerhaften Polarisierung der verfügbaren Einkommen? ", in Wochenbericht des DIW 10, S. 101-108.

Grömling, Michael; Lichtblau, Karl; Weber, Alexander 1998. Industrie und Dienstleistungen im Zeitalter der Globalisierung. Köln: Institut der Deutschen Wirtschaft.

Hamnett, Chris 2003. Unequal city: London in the global arena. London: Routledge.

Harrison, Barry; Bluestone, Bennett 1988. The great u-turn, corporate restructuring and the polarizing of America. New York: Basic Books.

Hauser, Richard 2003. "Die Entwicklung der Einkommens- und Vermögensverteilung in Deutschland - ein Überblick «, in Informationen zur Raumentwicklung 3-4, S. 111-124.

Häußermann, Hartmut; Siebel, Walter 1995. Dienstleistungsgesellschaften. Frankfurt a. M.: Suhrkamp.

Heitmeyer, Wolfgang; Dollase, Rainer; Backes, Otto. Hrsg. 1998. Die Krise der Städte. Analysen zu den Folgen desintegrativer Stadtentwicklung für das ethnisch-kulturelle Zusammenleben. Frankfurt a. M.: Suhrkamp.

Kalmbach, Peter; Franke, Reiner; Knottenbauer, Karin; Krämer, Hagen 2005. Die Interdependenz von Industrie und Dienstleistungen - Zur Dynamik eines komplexen Beziehungsgeflechts. Berlin: edition sigma.

Kroll-Schretzenmayr, Martina; Ritterhoff, Frank; Siebel, Walter 2005. »Wie global ist die Weltstadthypothese? Soziale und räumliche Polarisierung in der europäischen Stadt. Das Beispiel Zürich «, in disP 4, S. 50-73.

Montalvo, Jose G.; Reynal-Querol, Marta 2005. »Ethnic polarization, potential conflict, and civil wars", in The American Economic Review 95, 3, S. 796-816. 
OECD 2011. Divided we stand. Why inequality keeps rising. http://www.oecd-ilibrary.org/soci al-issues-migration-health/the-causes-of-growing-inequalities-in-oecd-countries_97892641 19536-en (Zugriff vom 15.07.2012).

Reynal-Querol, Marta 2002. »Ethnicity, political systems, and civil war ", in Journal of Conflict Resolution 46, 1, S. 29-54.

Sassen, Saskia 1994. Cities in a world economy. Sociology for a new century. Thousand Oaks, London, New Delhi: Pine Forge Press.

Sassen, Saskia 2001. The global city. New York, London, Tokyo: Princeton University Press.

Sassen, Saskia 2002. "Global Cities und ihr Hinterland ", in Brandenburg 2025 in der Mitte Europas, Band 1, hrsg. v. Zöpel, Christoph, S. 13-15. Potsdam.

Scharpf, Fritz W. 1986. "Strukturen der postindustriellen Gesellschaft«, in Soziale Welt 37, 1, S. 3-24.

Schwarze, Johannes 2003. »Using panel data on income satisfaction to estimate equivalence scale elasticity ", in The Review of Income and Wealth 49, 3, S. 359-372

Vaattovaara, Mari; Kortteinen, Matti 2003. »Beyond polarisation versus professionalisation? A case study of the development of Helsinki region, Finland ", in Urban Studies 40, S. 2127-2145.

Wagner, Gert G.; Frick, Joachim R.; Schupp, Jürgen 2007. »The German Socio-Economic Panel Study (SOEP) - scope, evolution and enhancements ", in Schmollers Jahrbuch (Journal of Applied Social Science Studies) 127, 1, S. 139-169.

Wessel, Terje 2000. "Social polarization and socioeconomic segregation in a welfare state: the case of Oslo", in Urban Studies 37, S. 1947-1967.

Wolfson, Michael C. 1994. »When inequalities diverge «, in The American Economic Review 84, 2, S. 353-358.

Wolfson, Michael C. 1997. "Divergent inequalities: theory and empirical results «, in Review of Income and Wealth 43, 4, S. 401-421. 


\section{Anhang}

Tabelle A1: Einkommenspolarisierung nach wirtschaftlichem Entwicklungstyp in Stadtregionen - Vergleich der Periode 2004-2006 zu 2000-2002

\begin{tabular}{|c|c|c|c|c|c|c|}
\hline \multicolumn{7}{|l|}{ Personenanteile } \\
\hline & \multicolumn{3}{|c|}{ Generelles Wachstum } & \multicolumn{3}{|c|}{ Tertiäres Wachstum } \\
\hline & $\begin{array}{l}2000- \\
2002\end{array}$ & $\begin{array}{l}2004- \\
2006\end{array}$ & $\begin{array}{l}\text { Ände- } \\
\text { rung }\end{array}$ & $\begin{array}{l}2000- \\
2002\end{array}$ & $\begin{array}{l}2004- \\
2006\end{array}$ & $\begin{array}{l}\text { Ände- } \\
\text { rung }\end{array}$ \\
\hline \multicolumn{7}{|c|}{ Personen in Haushalten mit ... } \\
\hline $\begin{array}{l}\text { niedrigen Einkommen } \\
\text { mittleren Einkommen } \\
\text { hohen Einkommen }\end{array}$ & $\begin{array}{l}14,7 \\
59,2 \\
26,1\end{array}$ & $\begin{array}{l}16,8 \\
56,5 \\
26,7\end{array}$ & $\begin{array}{r}14,3 \% \\
-4,6 \% \\
2,3 \%\end{array}$ & $\begin{array}{l}13,7 \\
65,4 \\
20,9\end{array}$ & $\begin{array}{l}14,9 \\
61,5 \\
23,5\end{array}$ & $\begin{array}{r}8,8 \% \\
-6 \% \\
12,4 \%\end{array}$ \\
\hline \multirow[t]{3}{*}{ Insgesamt } & 100,0 & 100,0 & & 100,0 & 99,9 & \\
\hline & \multicolumn{3}{|c|}{ Industrielle Schrumpfung } & \multicolumn{3}{|c|}{ Generelle Schrumpfung } \\
\hline & $\begin{array}{l}2000- \\
2002\end{array}$ & $\begin{array}{l}2004- \\
2006\end{array}$ & $\begin{array}{l}\text { Ände- } \\
\text { rung }\end{array}$ & $\begin{array}{l}2000- \\
2002\end{array}$ & $\begin{array}{l}2004- \\
2006\end{array}$ & $\begin{array}{l}\text { Ände- } \\
\text { rung }\end{array}$ \\
\hline \multicolumn{7}{|c|}{ Personen in Haushalten mit ... } \\
\hline $\begin{array}{l}\text { niedrigen Einkommen } \\
\text { mittleren Einkommen } \\
\text { hohen Einkommen }\end{array}$ & $\begin{array}{l}16,8 \\
65,3 \\
17,9\end{array}$ & $\begin{array}{l}20,3 \\
58,4 \\
21,3\end{array}$ & $\begin{array}{r}20,8 \% \\
-10,6 \% \\
19,0 \%\end{array}$ & $\begin{array}{l}26,1 \\
60,0 \\
13,9\end{array}$ & $\begin{array}{l}29,7 \\
57,1 \\
13,1\end{array}$ & $\begin{array}{l}13,8 \% \\
-4,8 \% \\
-5,8 \%\end{array}$ \\
\hline Insgesamt & 100,0 & 100,0 & & 100,0 & 99,9 & \\
\hline \multicolumn{7}{|l|}{ Mittlere Einkommen } \\
\hline & \multicolumn{3}{|c|}{ Generelles Wachstum } & \multicolumn{3}{|c|}{ Tertiäres Wachstum } \\
\hline & $\begin{array}{l}2000- \\
2002\end{array}$ & $\begin{array}{l}2004- \\
2006\end{array}$ & $\begin{array}{c}\text { Ände- } \\
\text { rung }\end{array}$ & $\begin{array}{l}2000- \\
2002\end{array}$ & $\begin{array}{l}2004- \\
2006\end{array}$ & $\begin{array}{c}\text { Ände- } \\
\text { rung }\end{array}$ \\
\hline \multicolumn{7}{|c|}{ Mittleres Einkommen von Personen in Haushalten mit ... } \\
\hline $\begin{array}{l}\text { niedrigen Einkommen } \\
\text { mittleren Einkommen } \\
\text { hohen Einkommen }\end{array}$ & $\begin{array}{r}628 \\
1.248 \\
2.462\end{array}$ & $\begin{array}{r}631 \\
1.222 \\
2.571\end{array}$ & $\begin{array}{r}0,5 \% \\
-2,1 \% \\
4,4 \%\end{array}$ & $\begin{array}{r}645 \\
1.263 \\
2.244\end{array}$ & $\begin{array}{r}626 \\
1.243 \\
2.373\end{array}$ & $\begin{array}{r}-2,9 \% \\
-1,6 \% \\
5,7 \%\end{array}$ \\
\hline \multirow{2}{*}{ hohen Einkommen } & \multicolumn{3}{|c|}{ Industrielle Schrumpfung } & \multicolumn{3}{|c|}{ Generelle Schrumpfung } \\
\hline & $\begin{array}{l}2000- \\
2002\end{array}$ & $\begin{array}{l}2004- \\
2006\end{array}$ & $\begin{array}{l}\text { Ände- } \\
\text { rung }\end{array}$ & $\begin{array}{l}2000- \\
2002\end{array}$ & $\begin{array}{l}2004- \\
2006\end{array}$ & $\begin{array}{l}\text { Ände- } \\
\text { rung }\end{array}$ \\
\hline \multicolumn{7}{|c|}{ Mittleres Einkommen von Personen in Haushalten mit ... } \\
\hline $\begin{array}{l}\text { niedrigen Einkommen } \\
\text { mittleren Einkommen } \\
\text { hohen Einkommen }\end{array}$ & $\begin{array}{r}629 \\
1.222 \\
2.378\end{array}$ & $\begin{array}{r}631 \\
1.203 \\
2.377\end{array}$ & $\begin{array}{r}0,3 \% \\
-1,6 \% \\
0,0 \%\end{array}$ & $\begin{array}{r}635 \\
1.222 \\
2.366\end{array}$ & $\begin{array}{r}622 \\
1.189 \\
2.310\end{array}$ & $\begin{array}{l}-2,0 \% \\
-2,7 \% \\
-2,4 \%\end{array}$ \\
\hline
\end{tabular}

Quelle: SOEP v 26, eigene Berechnungen. 
Tabelle A2: Polarisationsmaße zur Einkommensentwicklung in Agglomerationsräumen der Perioden 2000-2002 und 2004-2006 und Bootstrap-Konfidenzintervalle

\begin{tabular}{|c|c|c|c|}
\hline \multicolumn{4}{|c|}{ Reynal-Querol-Index: Periode 2000-2002 } \\
\hline & untere Grenze & PRQ & obere Grenze \\
\hline Wachstum generell & 0,84 & 0,85 & 0,86 \\
\hline Wachstum tertiär & 0,77 & 0,79 & 0,82 \\
\hline Schrumpfung industriell & 0,78 & 0,79 & 0,80 \\
\hline Schrumpfung generell & 0,83 & 0,84 & 0,86 \\
\hline \multicolumn{4}{|c|}{ Reynal-Querol-Index: Periode 2004-2006 } \\
\hline & untere Grenze & PRQ & obere Grenze \\
\hline Wachstum generell & 0,85 & 0,86 & 0,87 \\
\hline Wachstum tertiär & 0,81 & 0,83 & 0,85 \\
\hline Schrumpfung industriell & 0,83 & 0,84 & 0,85 \\
\hline Schrumpfung generell & 0,86 & 0,87 & 0,88 \\
\hline \multicolumn{4}{|c|}{ Esteban-Ray-Index: Periode 2000-2002 } \\
\hline & untere Grenze & PER & obere Grenze \\
\hline Wachstum generell & 0,117 & 0,120 & 0,123 \\
\hline Wachstum tertiär & 0,098 & 0,101 & 0,105 \\
\hline Schrumpfung industriell & 0,108 & 0,110 & 0,113 \\
\hline Schrumpfung generell & 0,107 & 0,110 & 0,113 \\
\hline \multicolumn{4}{|c|}{ Esteban-Ray-Index: Periode 2004-2006 } \\
\hline & untere Grenze & PER & obere Grenze \\
\hline Wachstum generell & 0,124 & 0,128 & 0,132 \\
\hline Wachstum tertiär & 0,109 & 0,114 & 0,119 \\
\hline Schrumpfung industriell & 0,113 & 0,115 & 0,118 \\
\hline Schrumpfung generell & 0,109 & 0,112 & 0,116 \\
\hline \multicolumn{4}{|c|}{ Esteban-Gardin-Ray-Index: Periode 2000-2002 } \\
\hline & untere Grenze & PEGR & obere Grenze \\
\hline Wachstum generell & 0,053 & 0,054 & 0,056 \\
\hline Wachstum tertiär & 0,045 & 0,046 & 0,048 \\
\hline Schrumpfung industriell & 0,049 & 0,050 & 0,051 \\
\hline Schrumpfung generell & 0,051 & 0,053 & 0,055 \\
\hline \multicolumn{4}{|c|}{ Esteban-Gardin-Ray-Index: Periode 2004-2006 } \\
\hline & untere Grenze & PEGR & obere Grenze \\
\hline Wachstum generell & 0,058 & 0,059 & 0,061 \\
\hline Wachstum tertiär & 0,050 & 0,051 & 0,054 \\
\hline Schrumpfung industriell & 0,053 & 0,055 & 0,056 \\
\hline Schrumpfung generell & 0,053 & 0,055 & 0,057 \\
\hline
\end{tabular}

Quelle: SOEP v 26, eigene Berechnungen. 
Zusammenfassung: Das einheitliche Modell von Stadtentwicklung, das auf Zunahme von Bevölkerung und Arbeitsplätzen sowie steigenden Realeinkommen beruht, hat sich mehr und mehr als unhaltbar erwiesen. Die Pfade der Stadtentwicklung sowie der Lebenslagen und Lebenschancen ihrer Bewohner sind unterschiedlicher denn je. Als Indiz für diese Entwicklung analysieren wir mit Hilfe der Individualdaten des Sozio-oekonomischen Panels (SOEP) die personelle Einkommensverteilung in Stadtregionen mit unterschiedlicher wirtschaftlicher Dynamik. Besonders deutlich nimmt danach die Einkommenspolarisierung in Stadtregionen zu, die durch einen starken Strukturwandel von der Industrie zu Dienstleistungen geprägt sind. Unsere empirische Arbeit bestätigt also den Einfluss der Deindustrialisierung auf die Einkommensverteilung, wie er Mitte der 1980er Jahre in den Städten der USA untersucht wurde. Mit einem breiten dynamischen Wirtschaftswachstum ist dagegen keine überdurchschnittliche Einkommenspolarisierung verbunden.

Stichworte: Einkommenspolarisierung, regionales Wachstum, Tertiarisierung, SOEP, Stadtpolitik

\title{
Economic dynamics of cities and income polarisation An empirical Study of German metropolitan areas
}

Summary: The model of urban development, which was based on the assumption of increasing population and jobs as well as increasing real incomes, has proven to be inadequate to reflect current conditions. Today, the processes of urban development and the living conditions and life chances of city dwellers differ more widely than ever before. In this study, we use individual data from the Socio-Economic Panel (SOEP) study as indicators for this development to examine the personal income distribution in urban regions affected by different economic dynamics. Income polarization is on the rise in urban areas that are experiencing a major structural shift from industry to services. Our empirical findings of German metropolitan areas confirm the influence of deindustrialization on the income distribution, similar to that found in examinations of various US states in the mid-1980 s. Dynamic, broadbased economic growth, however, is not associated with above-average income polarization.

Keywords: Income polarisation, regional development, tertiarisation, SOEP, city politics

\author{
Autoren \\ Dr. Jan Goebel \\ Leiter des Forschungsdatenzentrums SOEP \\ Deutsches Institut für Wirtschaftsforschung (DIW) \\ Mohrenstr. 58, 10117 Berlin \\ jgoebel@diw.de \\ Prof. Dr. Martin Gornig \\ Honorarprofessor für Stadt- und Regionalökonomie an der TU Berlin \\ Leiter der Abteilung Innovation, Industrie, Dienstleistungen \\ Deutsches Institut für Wirtschaftsforschung (DIW) \\ Mohrenstr. 58, 10117 Berlin \\ mgornig@diw.de
}

Hartmut Häußermann war Professor für Stadt- und Regionalsoziologie an der Humboldt-Universität zu Berlin. 